\title{
Rectal corticosteroids versus alternative treatments in ulcerative colitis: a meta-analysis
}

\author{
J K Marshall, E J Irvine
}

\begin{abstract}
Background-Clear strategies to optimise the use of corticosteroids in ulcerative colitis are lacking.

Aim-A meta-analysis was undertaken to examine critically the role of rectal corticosteroids in the management of active distal ulcerative colitis.

Methods-All reported randomised controlled trials were retrieved by searching the Medline and EMBASE databases and the bibliographies of relevant studies. Trials which met inclusion criteria were assessed for scientific rigour. Data were extracted by two independent observers according to predetermined criteria.

Results-Of 83 trials retrieved, 33 met inclusion criteria. Pooled odds ratios (POR) showed conventional rectal corticosteroids and rectal budesonide to be clearly superior to placebo. In seven trials, rectal 5-aminosalicylic acid (5-ASA) was significantly better than conventional rectal corticosteroids for inducing remission of symptoms, endoscopy, and histology with POR of 2.42 (95\% confidence interval (CI) 1.72-3.41), 1.89 (95\% CI 1.29-2.76), and 2.03 (95\% CI 1.28-3.20), respectively. Rectal budesonide was of comparable efficacy to conventional corticosteroids but produced less endogenous cortisol suppression. Side effects, although inconsistently reported, were generally minor. A cost comparison of rectal preparations showed 5-ASA to be less expensive than corticosteroids.

Conclusions-Rectal 5-ASA is superior to rectal corticosteroids in the management of distal ulcerative colitis.

(Gut 1997; 40: 775-781)
\end{abstract}

Keywords: ulcerative colitis, corticosteroids, therapy, topical administration, enema, suppository.

Oral corticosteroids have been a well-accepted treatment for active ulcerative colitis since Truelove et al reported the efficacy of oral hydrocortisone over 40 years ago. ${ }^{1}$ However, their long term use may be limited and patient compliance diminished by potential adverse effects. Administration of a corticosteroid liquid enema was first suggested to be efficacious in distal ulcerative colitis in $1956 .{ }^{2}$ The proven efficacy of direct drug delivery to the site of inflammation has since led to widespread acceptance of rectal corticosteroid therapy. ${ }^{3}$

The ability of a rectal preparation to achieve a proximal distribution is determined by the type of vehicle. Liquid enemas can deliver medication consistently to the splenic flexure, ${ }^{4-9}$ and a larger volume seems to allow more proximal delivery. ${ }^{10} 11$ Rectal foam disseminates medication to the rectum and distal descending colon, ${ }^{12-16}$ whereas suppositories coat only the rectum. ${ }^{17} 18$

Although studies of rectally administered corticosteroids have reported fewer systemic adverse effects than with oral preparations, plasma concentrations of prednisolone were similar after administration of identical oral or rectal doses. ${ }^{19} 20$ Suppression of the hypothalamic-pituitary-adrenal axis in association with rectal therapy has also been shown. ${ }^{21-26}$

Newer topically active corticosteroids such as tixocortol, beclomethasone, prednisolone metasulphabenzoate, and budesonide, with restricted absorption or rapid hepatic metabolism have been developed to reduce the adverse effects associated with conventional corticosteroids. ${ }^{27} 28$

To examine critically the role of rectal corticosteroids in the treatment of active distal ulcerative colitis, we performed a meta-analysis of all reported randomised controlled trials.

\section{Methods}

Relevant clinical trials were identified by searching the Medline database from 1966 to 1996 and the EMBASE database from 1985 to 1996, using the MeSH terms "inflammatory bowel disease", "therapy", and "topical administration", "enema", or "suppository". Bibliographies of all relevant studies and recent review articles were scanned to identify further citations. Each paper was assessed by two independent observers (JKM, EJI) according to predetermined inclusion criteria. Studies were accepted if patients had active ulcerative colitis with a documented disease margin distal to the splenic flexure on radiographic studies or less than $60 \mathrm{~cm}$ from the anal verge at flexible sigmoidoscopy or colonoscopy. We required that patients had been randomly assigned to two or more treatment groups, with rectal corticosteroids in at least one treatment arm and a symptom score as one of the main outcome criteria. The minimum duration of therapy permitted was two weeks.

Trials which met the inclusion criteria were then evaluated quantitatively for scientific rigour using a 30 point scoring system. ${ }^{29}$ Disagreements in scoring between the two observers were settled by consensus.

Data were extracted from each report using a predefined format. Recorded data points included the number of patients enrolled, number completing the study, sex, and disease distribution. The proportion of patients that improved or attained remission, or both, by 
symptomatic, endoscopic, and histological criteria were recorded from each trial using an intention to treat principle. The dose, frequency, duration, and formulation (enema, suppository or foam) of treatments were noted, as were any reported adverse effects of therapy.

We anticipated that the definitions of "improvement" and "remission" would vary considerably among the papers accepted. To overcome the problems of standardising endpoints, provided that an adequate definition of improvement or remission was offered, the authors' criteria for these outcomes were respected. All clinical symptom scores included stool frequency and rectal bleeding.

Studies were grouped for analysis according to the type of corticosteroid and control therapies. Corticosteroid doses were also converted to a hydrocortisone dose equivalent to permit testing for a dose response relation. ${ }^{30}$ The statistical analysis was conducted using the method of DerSimonian and Laird. ${ }^{31}$ An odds ratio for each trial and a common odds ratio for each group with $95 \%$ confidence intervals (CI) were calculated according to Mantel-Haenszel. Unless otherwise stated, odds ratios below 1 favoured corticosteroid treatment, whereas odds radios above 1 favoured the alternative treatment. Continuous data points were pooled and compared using a weighted mean difference. ${ }^{32}$ Homogeneity within groups of trials was confirmed using the Breslow-Day test. ${ }^{33}$ Overall response rates for each drug were calculated by dividing the total number of patients reaching an endpoint by the total number of patients treated.

\section{Results}

ACCEPTANCE AND VALIDITY SCORING

In total, 83 relevant trials were identified by the search, of which 33 met our inclusion criteria. Reasons for excluding a trial included: lack of randomisation (36 trials), inclusion of patients with disease proximal to the splenic flexure (29 trials), lack of a predefined symptom score (nine trials), duplicate reporting of data (three trials), and inclusion of patients with Crohn's colitis (one trial).

Table I summarises the characteristics of the 33 trials accepted for analysis. The median validity score (out of 30) was 21 (range 9-26).

RESPONSE RATES

Table II lists the response rates for all treatments and placebo. Among patients receiving conventional rectal corticosteroids (hydrocortisone, prednisolone, or betamethasone), pooled improvement rates by symptomatic, endoscopic, and histological criteria were $77 \%$, $66 \%$, and $52 \%$, whereas remission rates were $45 \%, 34 \%$, and $29 \%$, respectively. The pooled response rates for the topically active corticosteroids (budesonide, beclomethasone, or prednisolone metasulphobenzoate) were similar: $73 \%$ for symptoms, $69 \%$ for endoscopy, and $55 \%$ for histology, whereas $46 \%, 31 \%$, and $23 \%$, respectively, attained remission. When corticosteroid doses were converted to their hydrocortisone equivalent, ${ }^{30}$ no dose response relation was observed for either conventional or topical formulations. Similarly, no correlation was apparent between duration of treatment and response rate.

Aminosalicylates (4-ASA or 5-ASA), which were used most frequently as a comparative treatment, produced improvement in symptoms, endoscopy, and histology in, respectively, $81 \%, 75 \%$, and $65 \%$ of patients. Remission with these endpoints was induced in $52 \%, 41 \%$, and $32 \%$ of patients, respectively.

Across four trials, $34 \%$ of patients taking placebo improved symptomatically, and $38 \%$ improved endoscopically. Remission rates by symptomatic and endoscopic criteria were $9 \%$ and $17 \%$.

RECTAL CORTICOSTEROIDS VERSUS PLACEBO

Two trials compared conventional rectal corticosteroids with placebo. ${ }^{34}{ }^{35}$ The combined results clearly favoured corticosteroids, with a pooled odds ratios (POR) for symptomatic and endoscopic improvement of 0.21 (95\% CI $0.07-0.71)$ and 0.27 (95\% CI $0.10-0.77)$, respectively. The PORs for symptomatic and endoscopic remission were 0.07 (95\% CI $0 \cdot 02-0.29)$ and $0.34(95 \%$ CI $0 \cdot 10-1 \cdot 20)$, respectively. Histological endpoints were not reported in these early trials.

One trial reported that $2.3 \mathrm{mg}$ budesonide enemas were superior to placebo for improvement of symptoms, endoscopy, and histology, ${ }^{36}$ whereas another found $2 \cdot 0 \mathrm{mg}$ or $8 \cdot 0$ $\mathrm{mg}$ daily superior to placebo in inducing combined symptomatic and endoscopic remission, with higher response rates at the larger dose. ${ }^{37}$

RECTAL VERSUS ORAL CORTICOSTEROIDS

Rectal hydrocortisone $100 \mathrm{mg}$ was compared with oral prednisolone $60 \mathrm{mg}$ daily in one trial which showed oral treatment to be better for symptomatic improvement and remission. ${ }^{39} \mathrm{~A}$ second trial compared low dose oral prednisolone $(7.5 \mathrm{mg}$ daily) with rectal prednisolone metasulphobenzoate $20 \mathrm{mg}$, and found rectal treatment to be more efficacious for inducing symptomatic improvement. ${ }^{38}$ Because of substantial differences in oral dose, these results were not pooled.

\section{RECTAL CORTICOSTEROIDS VERSUS RECTAL}

5-ASA

Seven accepted trials compared rectal corticosteroids with rectal 5-ASA. ${ }^{40-46}$ The total daily dose of 5-ASA ranged from 1 to $4 \mathrm{~g}$, whereas the hydrocortisone equivalent dose of corticosteroids ranged from 100 to $356 \mathrm{mg}$. One trial compared a hydrocortisone foam with a 5-ASA suppository, ${ }^{43}$ whereas another compared hydrocortisone foam with 5-ASA foam. ${ }^{45}$ All other trials compared liquid enema preparations of equal volume. Pooled odds ratios for symptomatic, endoscopic, and histological improvement among the trials reporting these data were $1.36(95 \% \mathrm{CI} 0 \cdot 88-2.09), 1.06$ 
TABLE I Characteristics of trials accepted for meta-analysis

\begin{tabular}{|c|c|c|c|c|c|}
\hline \multicolumn{2}{|c|}{ Reference Author (year) } & Medication (dose and frequency) ${ }^{\star}$ & $\begin{array}{l}\text { Duration } \\
\text { (days) }\end{array}$ & $\begin{array}{l}\text { Number of } \\
\text { patients }\end{array}$ & $\begin{array}{l}\text { Validity score } \\
(\text { maximum }=30)\end{array}$ \\
\hline \multicolumn{6}{|c|}{ A Rectal corticosteroids $\mathrm{v}$ placebo } \\
\hline 34 & Lennard-Jones et al (1962) & $\begin{array}{l}\text { Prednisolone ( } 5 \text { mg SUPP od) } \\
\text { Placebo }\end{array}$ & 21 & 39 & 16 \\
\hline 35 & Watkinson (1958) & $\begin{array}{l}\text { Hydrocortisone }(100 \mathrm{mg} / 100 \mathrm{ml} \text { od }) \\
\text { Placebo }\end{array}$ & 15 & 19 & 17 \\
\hline \multicolumn{6}{|c|}{ B Rectal budesonide $\mathrm{v}$ placebo } \\
\hline 36 & Danielsson et al (1992) & $\begin{array}{l}\text { Budesonide }(2.3 \mathrm{mg} / 115 \mathrm{ml} \mathrm{od}) \\
\text { Placebo }\end{array}$ & 28 & 41 & 23 \\
\hline 37 & Hanauer and Robinson (1995) $†$ & $\begin{array}{l}\text { Budesonide }(0.5 \mathrm{mg} \text { or } 2.0 \mathrm{mg} \text { or } 8.0 \mathrm{mg} \text { od }) \\
\text { Placebo }\end{array}$ & 42 & 233 & 12 \\
\hline \multicolumn{6}{|c|}{ C Rectal $\mathrm{v}$ oral corticosteroids } \\
\hline 38 & Hamilton et al (1984) & $\begin{array}{l}\text { Prednisolone metasulphoenzoate ( } 20 \mathrm{mg} \text { od }) \\
\text { Prednisolone }(7.5 \mathrm{mg} \text { ORAL od })\end{array}$ & 14 & 36 & 17 \\
\hline 39 & Lennard-Jones et al (1960) & $\begin{array}{l}\text { Hydrocortisone }(100 \mathrm{mg} / 150 \mathrm{ml}) \text { od } \\
\text { Prednisone (variable dose ORAL od) } \\
\text { Salazopyrin (4 g ORAL od) }\end{array}$ & 21 & 60 & 15 \\
\hline \multicolumn{6}{|c|}{ D Rectal corticosteroids $\mathrm{v}$ rectal $5-A S A$} \\
\hline 40 & Bianchi Porro et al (1995) & $\begin{array}{l}\text { Hydrocortisone }(100 \mathrm{mg} / 60 \mathrm{ml} \text { od }) \\
5 \text {-ASA }(1 \mathrm{~g} / 100 \mathrm{ml} \mathrm{od})\end{array}$ & 21 & 52 & 23 \\
\hline 41 & Campieri et al (1981) & $\begin{array}{l}\text { Hydrocortisone }(100 \mathrm{mg} / 100 \mathrm{ml} \mathrm{od}) \\
5 \text {-ASA }(4 \mathrm{~g} / 100 \mathrm{ml} \mathrm{od})\end{array}$ & 15 & 86 & 19 \\
\hline 42 & Danish 5-ASA Group (1987) & $\begin{array}{l}\text { Prednisolone }(25 \mathrm{mg} / 100 \mathrm{ml} \mathrm{od}) \\
5 \text {-ASA }(1 \mathrm{~g} / 100 \mathrm{ml} \mathrm{od})\end{array}$ & 14 & 123 & 25 \\
\hline 43 & Farup et al (1995) & $\begin{array}{l}\text { Hydrocortisone }(178 \mathrm{mg} / 60 \mathrm{ml} \text { FOAM bid }) \\
\text { 5-ASA }(0.5 \mathrm{~g} \text { SUPP bid })\end{array}$ & 28 & 79 & 23 \\
\hline 44 & Friedman et al (1986) & $\begin{array}{l}\text { Hydrocortisone }(100 \mathrm{mg} / 100 \mathrm{ml} \mathrm{od}) \\
5 \text {-ASA }(4 \mathrm{~g} / 100 \mathrm{ml} \mathrm{od})\end{array}$ & 21 & 18 & 24 \\
\hline 45 & Lee et al (1996) & $\begin{array}{l}\text { Prednisolone }(20 \mathrm{mg} / 30 \mathrm{ml} \text { FOAM od }) \\
\text { 5-ASA }(2 \mathrm{~g} / 120 \mathrm{ml} \text { FOAM od })\end{array}$ & 28 & 295 & 25 \\
\hline 46 & Mulder et al (1988) & $\begin{array}{l}\text { Prednisolone }(30 \mathrm{mg} / 40 \mathrm{ml} \mathrm{od}) \\
5-A S A(3 \mathrm{~g} / 40 \mathrm{ml} \mathrm{od})\end{array}$ & 28 & 29 & 24 \\
\hline \multicolumn{6}{|c|}{$\mathrm{E}$ Rectal corticosteroids $\mathrm{v}$ rectal $4-A S A$} \\
\hline 47 & O'Donnell et al (1992) & $\begin{array}{l}\text { Prednisolone }(20 \mathrm{mg} / 50 \mathrm{ml} \mathrm{od}) \\
\text { 4-ASA }(2 \mathrm{~g} / 50 \mathrm{ml} \mathrm{od})\end{array}$ & 42 & 45 & 24 \\
\hline 48 & Sharma et al (1992) & $\begin{array}{l}\text { Prednisolone }(20 \mathrm{mg} / 60 \mathrm{ml} \text { od }) \\
\text { 4-ASA }(2 \mathrm{~g} / 60 \mathrm{ml} \mathrm{od})\end{array}$ & 28 & 40 & 26 \\
\hline \multicolumn{6}{|c|}{ F Rectal corticosteroids $\mathrm{v}$ rectal budesonide } \\
\hline 24 & Bianchi Porro et al (1994) & $\begin{array}{l}\text { Methylprednisolone }(20 \mathrm{mg} / 100 \mathrm{ml} \text { od }) \\
\text { Budesonide }(2 \mathrm{mg} / 100 \mathrm{ml} \mathrm{od})\end{array}$ & 28 & 88 & 21 \\
\hline 25 & Danielsson et al (1987) & $\begin{array}{l}\text { Prednisolone }(31 \cdot 25 \mathrm{mg} / 100 \mathrm{ml} \text { od }) \\
\text { Budesonide }(2 \mathrm{mg} / 100 \mathrm{ml} \mathrm{od})\end{array}$ & 28 & 64 & 20 \\
\hline 26 & Danish Budesonide Study Group (1991) & $\begin{array}{l}\text { Prednisolone }(25 \mathrm{mg} / 100 \mathrm{ml} \text { od }) \\
\text { Budesonide }(1 \text { or } 2 \text { or } 4 \mathrm{mg} / 100 \mathrm{ml} \mathrm{od})\end{array}$ & 14 & 139 & 19 \\
\hline 49 & Lofberg et al (1994) & $\begin{array}{l}\text { Prednisolone }(31.25 \mathrm{mg} / 125 \mathrm{ml} \mathrm{od}) \\
\text { Budesonide }(2.3 \mathrm{mg} / 115 \mathrm{ml} \mathrm{od})\end{array}$ & 56 & 100 & 24 \\
\hline 50 & Tarpila et al (1994) & $\begin{array}{l}\text { Hydrocortisone }(125 \mathrm{mg} / 125 \mathrm{ml} \text { od }) \\
\text { Budesonide }(2 \cdot 3 \mathrm{mg} / 115 \mathrm{ml} \mathrm{od})\end{array}$ & 28 & 72 & 20 \\
\hline \multicolumn{6}{|c|}{$\mathrm{G}$ Rectal budesonide $\mathrm{v}$ rectal 5- $A S A$} \\
\hline 51 & Lamers et al (1991)† & $\begin{array}{l}\text { Budesonide }(2 \mathrm{mg} / 100 \mathrm{ml} \text { od }) \\
5 \text {-ASA }(4 \mathrm{~g} / 60 \mathrm{ml} \text { od })\end{array}$ & 28 & 62 & 13 \\
\hline 52 & Lemann et al (1995) & $\begin{array}{l}\text { Budesonide }(2 \mathrm{mg} / 115 \mathrm{ml} \text { od }) \\
\text { 5-ASA }(1 \mathrm{~g} / 100 \mathrm{ml} \mathrm{od})\end{array}$ & 28 & 92 & 22 \\
\hline \multicolumn{6}{|c|}{ H Other } \\
\hline 53 & Cobden et al (1991) & $\begin{array}{l}\text { Prednisolone metabenzoate }(20 \mathrm{mg} / 100 \mathrm{ml} \text { bid }) \\
\text { 5-ASA }(0 \cdot 8 \mathrm{~g} \text { ORAL qid })\end{array}$ & 28 & 37 & 26 \\
\hline 54 & Grace et al (1987) & $\begin{array}{l}\text { Prednisolone }(20 \mathrm{mg} / 100 \mathrm{ml} \text { od }) \\
\text { Sodium cromoglycate }(600 \mathrm{mg} / 100 \mathrm{ml} \mathrm{od})\end{array}$ & 56 & 70 & 18 \\
\hline 55 & Halpern et al (1991) $\ddagger$ & $\begin{array}{l}\text { Beclomethasone dipropionate }(0.5 \mathrm{mg} / 100 \mathrm{ml} \mathrm{od}) \\
\text { Betamethasone phosphate }(5 \mathrm{mg} / 100 \mathrm{ml} \text { od })\end{array}$ & 28 & 40 & 22 \\
\hline 56 & Hanauer et al (1986)† & $\begin{array}{l}\text { Tixocortol pivalate }(250 \mathrm{mg} \text { od }) \\
\text { Hydrocortisone }(100 \mathrm{mg} \mathrm{od})\end{array}$ & 21 & 125 & 9 \\
\hline 57 & Lennard-Jones (1971) & $\begin{array}{l}\text { Betamethasone valerate }(5 \mathrm{mg} / 100 \mathrm{ml} \text { od }) \\
\text { Prednisolone }(20 \mathrm{mg} / 100 \mathrm{ml} \mathrm{od})\end{array}$ & 28 & 105 & 17 \\
\hline 58 & Mulder et al (1989) & $\begin{array}{l}\text { Beclomethasone diproprionate }(2 \text { or } 3 \mathrm{mg} / 40 \mathrm{ml} \text { od) } \\
\text { Prednisolone ( } 30 \mathrm{mg} / 40 \mathrm{ml} \text { od })\end{array}$ & 28 & 25 & 23 \\
\hline 59 & Mulder et al (1994)† & $\begin{array}{l}\text { Beclomethasone dipropionate }(3 \mathrm{mg} / 100 \mathrm{ml} \text { od }) \\
\text {-ASA }(1 \mathrm{~g} / 100 \mathrm{ml} \text { od) } \\
\text { Beclomethasone and } 5 \text {-ASA }(3 \mathrm{mg} / 100 \mathrm{ml} \text { and } 1 \mathrm{~g} / 100 \mathrm{ml} \text { od) }\end{array}$ & 28 & 60 & 13 \\
\hline 60 & Riley et al (1989) & $\begin{array}{l}\text { Prednisolone metasulphobenzoate }(20 \mathrm{mg} / 100 \mathrm{ml} \text { od }) \\
\text { Sucralfate }(4 \mathrm{~g} / 100 \mathrm{ml} \text { od })\end{array}$ & 28 & 44 & 23 \\
\hline 61 & Ruddell et al (1980) & $\begin{array}{l}\text { Hydrocortisone }(100 \mathrm{mg} / 60 \mathrm{ml} \text { bid }) \\
\text { Hydrocortisone }(100 \mathrm{mg} / 5 \mathrm{ml} \text { FOAM bid })\end{array}$ & 14 & 30 & 16 \\
\hline 62 & van der Heide et al (1988) & $\begin{array}{l}\text { Beclomethasone dipropionate }(1 \mathrm{mg} / 40 \mathrm{ml} \text { od }) \\
\text { Prednisolone }(30 \mathrm{mg} / 40 \mathrm{ml} \mathrm{od})\end{array}$ & 28 & 18 & 24 \\
\hline 63 & van Outryve et al (1996)† & $\begin{array}{l}\text { Ridogrel }(300 \mathrm{mg} / 40 \mathrm{ml} \mathrm{od}) \\
\text { Prednisolone }(30 \mathrm{mg} / 40 \mathrm{ml} \text { od })\end{array}$ & 28 & 40 & 17 \\
\hline
\end{tabular}

*Medication in enema format unless otherwise stated. †Abstract only. $¥$ Total 32 patients with 40 treatment courses. SUPP=suppository; od=once daily; bid=twice daily; qid=four times daily.

(95\% CI $0.61-1 \cdot 85)$, and $2 \cdot 27$ (95\% CI $1 \cdot 22-4 \cdot 27)$, with results clearly favouring 5-ASA only for histology (Figs 1 and 3). Using the stricter outcome of disease remission, 5-ASA was significantly better for all three criteria with PORs of $2 \cdot 42(95 \%$ CI $1 \cdot 72-3 \cdot 41)$ for symptoms, $1.89(1 \cdot 29-2 \cdot 76)$ for endoscopy, and $2.03(95 \%$ CI $1 \cdot 28-3 \cdot 20)$ for histology (Figs 2 and 3 ). When the two trials using foam preparations ${ }^{43}{ }^{45}$ were excluded from the analysis, recalculated PORs for remission endpoints still favoured 5-ASA significantly, despite wider confidence intervals as a result of the smaller sample size.

A single trial compared 5-ASA and beclomethasone enemas alone versus a combined 5-ASA/beclomethasone enema. ${ }^{59}$ The combination surpassed monotherapy in inducing symptomatic or endoscopic improvement $(\mathrm{p}<0 \cdot 05)$. 
TABLE II Pooled response rates for rectal preparations (all trials)

\begin{tabular}{|c|c|c|c|c|c|c|}
\hline \multirow[b]{3}{*}{ Medication } & \multicolumn{6}{|c|}{ Response rate across all trials (ratio, percentage) } \\
\hline & \multicolumn{3}{|l|}{ Improvement } & \multicolumn{3}{|l|}{ Remission } \\
\hline & Symptomatic & Endoscopic & Histologic & Symptomatic & Endoscopic & Histological \\
\hline $\begin{array}{l}\text { Conventional corticosteroids } \\
\text { Hydrocortisone } \\
\text { Prednisolone } \\
\text { Methylprednisolone } \\
\text { Betamethasone } \\
\text { Pooled }\end{array}$ & $\begin{array}{c}58 / 94(62 \%) \\
272 / 344(79 \%) \\
28 / 44(59 \%) \\
61 / 71(86 \%) \\
428 / 553(77 \%)\end{array}$ & $\begin{array}{c}61 / 109(56 \%) \\
146 / 196(74 \%) \\
20 / 44(45 \%) \\
18 / 20(90 \%) \\
245 / 369(66 \%)\end{array}$ & $\begin{array}{c}71 / 137(52 \%) \\
73 / 144(51 \%) \\
20 / 44(45 \%) \\
16 / 20(80 \%) \\
180 / 345(52 \%)\end{array}$ & $\begin{array}{c}50 / 119(42 \%) \\
149 / 338(44 \%) \\
16 / 44(36 \%) \\
40 / 71(56 \%) \\
255 / 572(45 \%)\end{array}$ & $\begin{array}{c}43 / 96(45 \%) \\
127 / 387(33 \%) \\
8 / 44(18 \%) \\
6 / 20(30 \%) \\
184 / 547(34 \%)\end{array}$ & $\begin{array}{c}14 / 51(27 \%) \\
83 / 262(32 \%) \\
6 / 44(14 \%) \\
10 / 20(50 \%) \\
113 / 377(30 \%)\end{array}$ \\
\hline $\begin{array}{l}\text { Topically active corticosteroids } \\
\text { Prednisolone metasulphobenzoate } \\
\text { Beclomethasone } \\
\text { Budesonide } \\
\text { Pooled }\end{array}$ & $\begin{array}{c}33 / 40(83 \%) \\
42 / 65(65 \%) \\
49 / 64(77 \%) \\
124 / 169(73 \%)\end{array}$ & $\begin{array}{c}15 / 22(68 \%) \\
44 / 65(68 \%) \\
163 / 237(69 \%) \\
222 / 324(69 \%)\end{array}$ & $\begin{array}{c}15 / 22(68 \%) \\
24 / 45(53 \%) \\
110 / 205(54 \%) \\
149 / 272(55 \%)\end{array}$ & $\begin{array}{c}15 / 22(68 \%) \\
9 / 20(45 \%) \\
53 / 137(39 \%) \\
77 / 169(46 \%)\end{array}$ & $\begin{array}{c}17 / 41(41 \%) \\
16 / 56(29 \%) \\
70 / 237(30 \%) \\
103 / 334(31 \%)\end{array}$ & $\begin{array}{c}10 / 22(45 \%) \\
5 / 20(25 \%) \\
26 / 137(19 \%) \\
41 / 179(23 \%)\end{array}$ \\
\hline $\begin{array}{l}\text { Aminosalicylates } \\
\text { 5-ASA } \\
\text { 4-ASA } \\
\text { Pooled } \\
\text { Placebo }\end{array}$ & $\begin{array}{c}230 / 282(82 \%) \\
37 / 47(79 \%) \\
267 / 329(81 \%) \\
18 / 53(34 \%)\end{array}$ & $\begin{array}{c}154 / 212(73 \%) \\
20 / 20(100 \%) \\
174 / 232(75 \%) \\
12 / 32(38 \%)\end{array}$ & $\begin{array}{c}93 / 141(66 \%) \\
29 / 47(62 \%) \\
122 / 188(65 \%)\end{array}$ & $\begin{array}{c}195 / 368(53 \%) \\
9 / 27(33 \%) \\
204 / 395(52 \%) \\
3 / 32(9 \%)\end{array}$ & $\begin{array}{c}140 / 384(36 \%) \\
18 / 20(90 \%) \\
158 / 404(39 \%) \\
5 / 32(17 \%)\end{array}$ & $\begin{array}{c}84 / 251(33 \%) \\
5 / 27(19 \%) \\
89 / 278(32 \%)\end{array}$ \\
\hline
\end{tabular}

RECTAL CORTICOSTEROIDS VERSUS RECTAL 4-ASA

Rectal corticosteroids were compared with rectal 4-ASA in two trials. ${ }^{47} 48$ Each compared 4-ASA $2 \mathrm{~g}$ with prednisolone $20 \mathrm{mg}$ (hydrocortisone dose equivalent $80 \mathrm{mg}$ ). POR for symptomatic improvement was 3.88 (95\% CI $1 \cdot 29-11 \cdot 64)$, favouring 4-ASA.

RECTAL CORTICOSTEROIDS VERSUS RECTAL BUDESONIDE

Five trials compared conventional rectal corticosteroids with rectal budesonide. ${ }^{24-26} 4950$ The

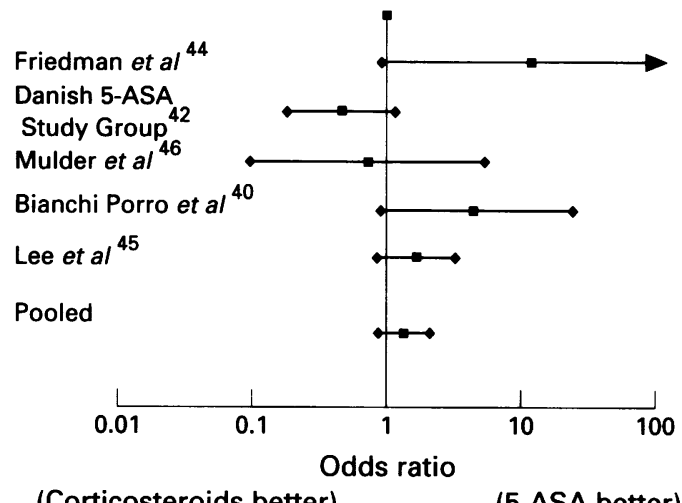

Figure 1: Symptomatic improvement: rectal corticosteroids $\mathbf{v}$ rectal 5-aminosalicylic acid (5- $A S A)$.

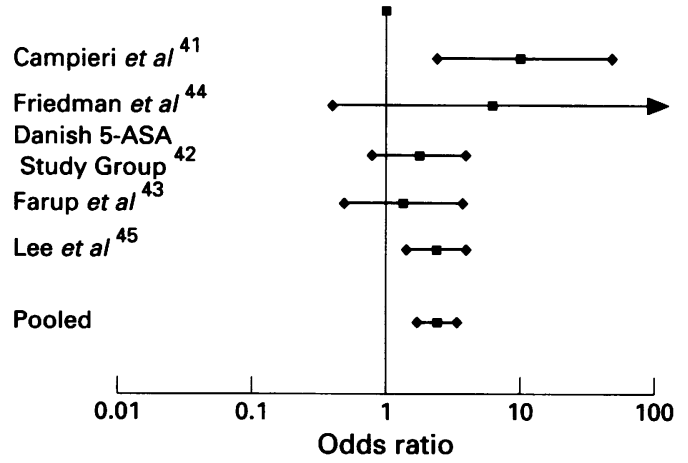

(Corticosteroids better)

(5-ASA better)

Figure 2: Symptomatic remission: rectal corticosteroids $\mathrm{v}$ rectal 5-aminosalicylic acid (5-ASA). budesonide dose ranged from 2.0 to $2.5 \mathrm{mg}$, whereas the corticosteroid dose ranged from 100 to $125 \mathrm{mg}$ of hydrocortisone equivalent. Data from one trial could not be extracted adequately for meta-analysis. ${ }^{26}$ One trial used a hydrocortisone foam..$^{50}$ All other medications were given as enemas.

The PORs for improvement by symptomatic, endoscopic, and histological criteria were $2 \cdot 08$ (95\% CI $0 \cdot 84-5 \cdot 14), 1 \cdot 40$ (95\% CI $0 \cdot 87-2 \cdot 25)$, and $1.23(95 \%$ CI $0 \cdot 80-1 \cdot 91)$, respectively (Fig 4). PORs for symptomatic, endoscopic, and histological remission were $0.85(95 \%$ CI $0.44-1 \cdot 63), 1 \cdot 14(95 \%$ CI $0 \cdot 69-1 \cdot 88)$, and $0 \cdot 68(95 \%$ CI $0 \cdot 28-1 \cdot 67)$. All confidence intervals included 1 .

RECTAL BUDESONIDE VERSUS RECTAL 5-ASA

Two trials comparing rectal budesonide with rectal 5-ASA were evaluated. ${ }^{51} 52$ Endoscopic improvement and remission data were reported in both trials, with a POR of $0.58(95 \%$ CI $0.27-1 \cdot 22)$ and $0.95(95 \%$ CI $0.43-2 \cdot 10)$, respectively, where an OR $<1$ favoured 5-ASA. In one of the trials 5-ASA exceeded budesonide for inducing symptomatic remission, with an OR of $0.41(95 \%$ CI $0 \cdot 18-0.94) .^{52}$ The other reported similar symptomatic remission rates in both treatment arms, but the data provided did not permit calculation of an $\mathrm{OR}^{51}$

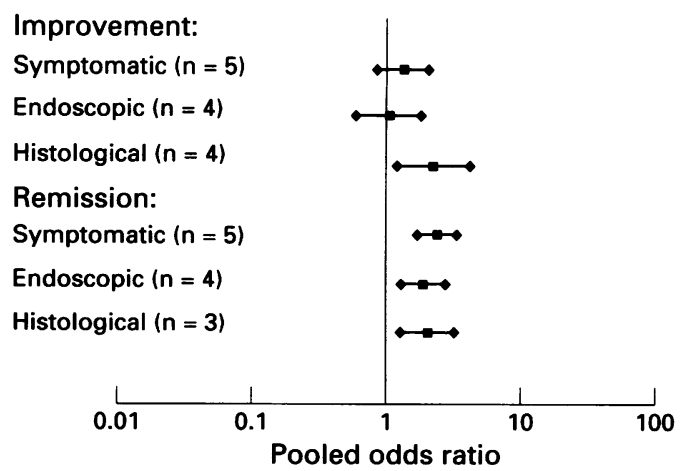

(Corticosteroids better)

(5-ASA better)

Figure 3: Pooled odds ratios for all outcomes: rectal corticosteroids $\mathrm{v}$ rectal 5-aminosalicylic acid (5- $A S A$ ). 


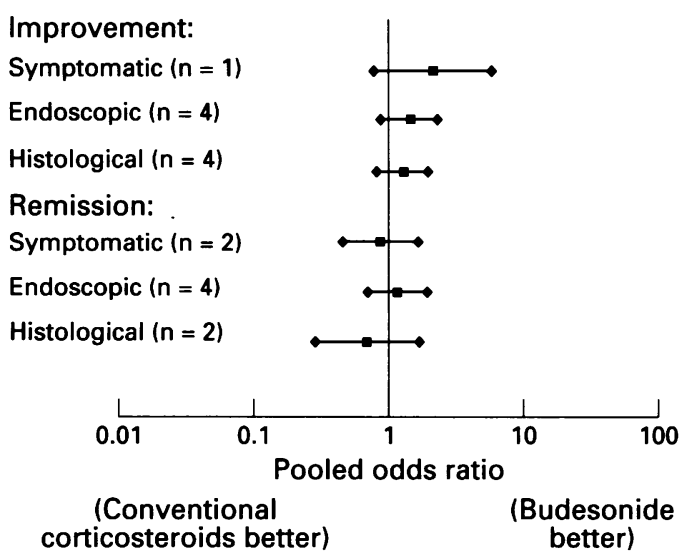

Figure 4: Pooled odds ratios for all outcomes: conventional rectal corticosteroids $\mathrm{v}$ rectal budesonide.

\section{ADVERSE EFFECTS OF RECTAL CORTI-}

COSTEROIDS

Adverse effects of treatment were inconsistently reported in the accepted trials. Nine of the 33 trials made no reference whatsoever to adverse effects, whereas a further 11 trials reported no drug related adverse effects in any treatment arm. Among the remaining 13 trials, seven dropouts for drug related effects were noted: four on 5-ASA, one on conventional corticosteroids, one on budesonide, and one

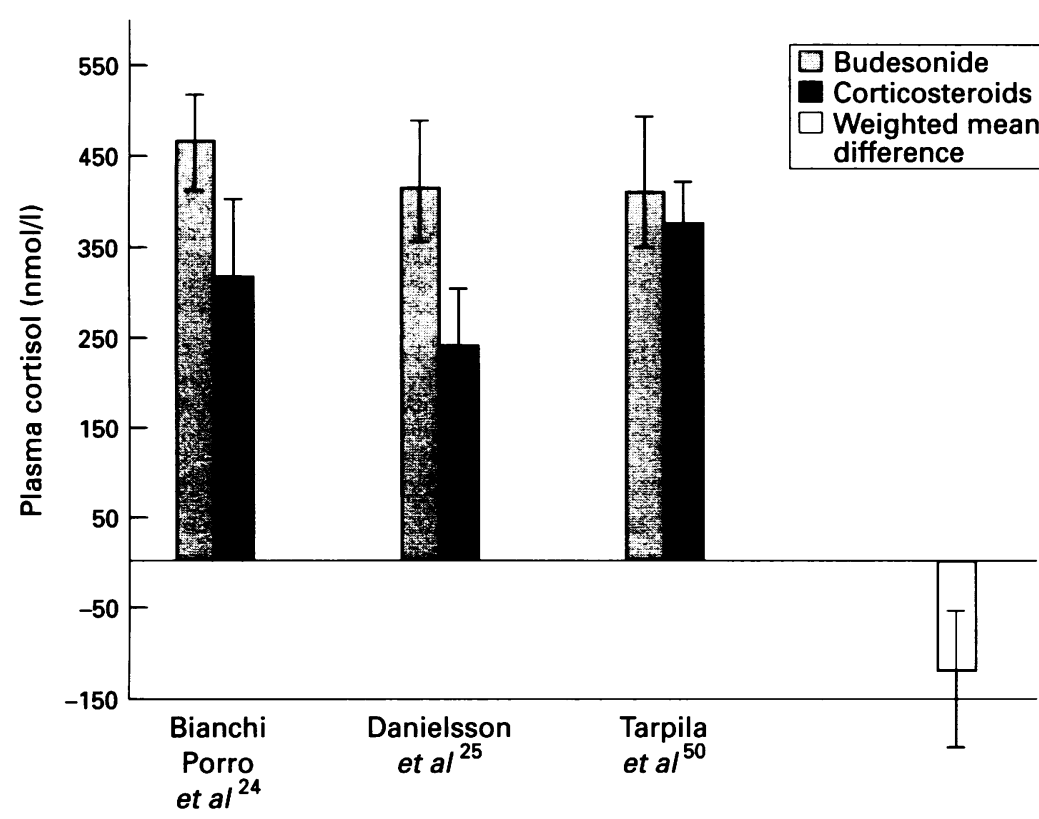

Figure 5: Plasma cortisol concentrations and weighted mean difference (with 95\% CI) after four weeks of treatment: conventional rectal corticosteroids $\mathrm{v}$ rectal budesonide.

TABLE III Cost of available rectal treatments (Chedoke-McMaster Hospital Pharmacy, Fuly 1996)

\begin{tabular}{|c|c|c|}
\hline Medication & $\begin{array}{l}\text { Unit cost } \\
\text { (Can S; USS; } €) \\
\text { excluding dispensing fee }\end{array}$ & $\begin{array}{l}\text { Cost of } 14 \text { day } \\
\text { course (Can \$; USS; } € \text { ) } \\
\text { excluding dispensing fee }\end{array}$ \\
\hline $\begin{array}{l}\text { Hydrocortisone enema } 100 \mathrm{mg} \text { (Cortenema) } \\
\text { Hydrocortisone foam } 80 \mathrm{mg} \\
\text { (Cortifoam } 14 \text { dose cannister) }\end{array}$ & $\begin{array}{c}6 \cdot 93 ; 4 \cdot 89 ; 3 \cdot 00 \\
83 \cdot 24 ; 58 \cdot 77 ; 36 \cdot 04\end{array}$ & $\begin{array}{l}97 \cdot 02 ; 68 \cdot 50 ; 42 \cdot 01 \\
83 \cdot 24 ; 58 \cdot 77 ; 36 \cdot 04\end{array}$ \\
\hline $\begin{array}{l}\text { Betamethasone enema } 5 \mathrm{mg} \text { (Betnesol) } \\
\text { Budesonide enema } 2 \cdot 3 \mathrm{mg} \text { (Entocort) } \\
\text { Tixocortol } 250 \mathrm{mg} \text { (Rectovalone) } \\
\text { 5-ASA enema } 4 \mathrm{~g} \text { (Salofalk) } \\
\text { 5-ASA enema } 2 \mathrm{~g} \text { (Salofalk) } \\
\text { 5-ASA enema } 2 \mathrm{~g} \text { (Quintasa) } \\
\text { 5-ASA enema } 1 \mathrm{~g} \text { (Quintasa) }\end{array}$ & $\begin{array}{l}8 \cdot 68 ; 6 \cdot 13 ; 3 \cdot 76 \\
9 \cdot 00 ; 6 \cdot 35 ; 3.90 \\
7 \cdot 95 ; 5 \cdot 61 ; 3 \cdot 44 \\
7 \cdot 08 ; 5 \cdot 00 ; 3.07 \\
4 \cdot 10 ; 2 \cdot 89 ; 1 \cdot 78 \\
4 \cdot 35 ; 3 \cdot 07 ; 1 \cdot 88 \\
3.97 ; 2 \cdot 80 ; 1 \cdot 72\end{array}$ & $\begin{array}{r}121 \cdot 52 ; 85 \cdot 79 ; 52 \cdot 62 \\
126 \cdot 00 ; 88 \cdot 96 ; 54 \cdot 56 \\
111 \cdot 30 ; 78 \cdot 58 ; 48 \cdot 19 \\
99 \cdot 12 ; 69 \cdot 98 ; 42 \cdot 92 \\
57 \cdot 40 ; 40 \cdot 52 ; 24 \cdot 85 \\
60 \cdot 90 ; 43 \cdot 00 ; 26 \cdot 37 \\
55 \cdot 58 ; 39 \cdot 24 ; 24 \cdot 07\end{array}$ \\
\hline
\end{tabular}

on 4-ASA. Other drug related adverse effects such as nausea, abdominal distension, fatigue, and perianal irritation were infrequent.

Overall, 10 trials reported hypothalamicpituitary-adrenal axis function before and after treatment. Three of these compared rectal budesonide with conventional rectal corticosteroids, noting mean cortisol concentrations after four weeks of treatment. ${ }^{25} 4950$ Cortisol concentrations were consistently higher, indicating lesser suppression, in the budesonide group than in the group receiving conventional corticosteroids (Fig 5). The weighted mean difference between pooled treatment arms was $119 \cdot 1 \mathrm{nmol} / 1$ (95\% CI 70.3-167.9), confirming that this difference was statistically significant. Another trial reported similar data using an analog scale which could not be pooled. ${ }^{26}$

COST COMPARISON

The costs for rectal steroid and 5-ASA enema and foam preparations available in Canada, in Canadian dollars, were obtained from the Chedoke-McMaster hospital pharmacy and are shown in Table III. Hydrocortisone $100 \mathrm{mg}$ and 5-ASA $4 \mathrm{~g}$ enemas are comparable in cost, whereas 5-ASA $1 \mathrm{~g}$ and $2 \mathrm{~g}$ enemas cost considerably less. Budesonide enemas are marginally more expensive than hydrocortisone liquid enema, and hydrocortisone foam costs slightly less. Methylprednisolone enemas currently are not available in Canada.

\section{Discussion}

This study confirms that rectal corticosteroids are an effective treatment for active distal ulcerative colitis, with a therapeutic gain over placebo of approximately $30 \%$, but suggests that rectal 5 -ASA is significantly more efficacious for inducing disease remission.

Placebo controlled data are becoming increasingly scarce in the recent literature, possibly because of the ethical concerns of treating patients with active ulcerative colitis with placebo. Two early placebo controlled trials $^{3463}$ confirmed the efficacy of conventional rectal corticosteroids for inducing improvement and remission by symptomatic and endoscopic criteria. Two recent trials ${ }^{36} 37$ also showed the topically acting corticosteroid budesonide to be superior to placebo using symptomatic, endoscopic, and histological endpoints. Our pooled placebo data demonstrated symptomatic and endoscopic improvement in $34 \%$ and $38 \%$, and symptomatic and endoscopic remission in $9 \%$ and $17 \%$ of patients, respectively. These findings were similar to those of another overview which suggested that placebo benefited $30 \%$ of patients and produced remission in $10 \%{ }^{64}$

Our meta-analysis suggests that rectal 5-ASA is as efficacious as rectal corticosteroids for improving disease and is better than rectal corticosteroids for inducing remission. Results were consistent, with similarly narrow confidence intervals, for symptomatic, endoscopic, or histological outcomes. The two trials which 
compared rectal 5-ASA with budesonide suggested that 5-ASA is at least as effective in producing disease improvement and remission. However, not all endpoints were noted.

Only two studies compared the role of rectal 4-ASA with corticosteroids, but supported a therapeutic gain in producing symptomatic improvement for 4-ASA.

Budesonide is a new topically active corticosteroid formulation with a high glucocorticoid receptor affinity, and significant first pass hepatic metabolism. The pooled results for accepted trials failed to demonstrate significant therapeutic benefit of either budesonide or conventional corticosteroids. However, budesonide caused significantly less endogenous cortisol suppression, based on pooled mean cortisol concentrations. Although these data are promising, no data have been reported regarding differences in steroid specific adverse effects, such as osteopenia or Cushingoid facies.

Other factors which may influence the efficacy of a treatment formulation include the proximal extent of the disease. Although our pooled data did not permit subgroup analysis, foam and suppositories did produce higher response rates in patients with more distal disease, ${ }^{43}$ supporting the findings of radiological and radionuclide studies of a more distal distribution of medication. ${ }^{12-18}$ Patient preference for foam or suppository preparations also may augment compliance, and hence the effectiveness of therapy. ${ }^{61}$ Similarly, the volume of enema or foam preparations may influence treatment distribution and efficacy. ${ }^{10}$ Although most trials used equivalent volumes in both treatment arms, Lee et $a l^{45}$ compared a $30 \mathrm{cc}$ prednisolone foam with a $120 \mathrm{cc} 5$-ASA foam, a bias which could favour the efficacy of 5-ASA. When these data were excluded from the pooled results, 5-ASA remained superior to corticosteroids in inducing remission, although confidence intervals were wider.

An important feature which potentially could confound results of this meta-analysis was duration of treatment, which ranged from 14 to 56 days among the trials accepted. Although pooled results of trial endpoints did not demonstrate a clear relation, individual trials which reported interim endpoints at different time intervals observed higher endoscopic and histological remission rates with prolonged treatment. ${ }^{25} 36424349-51$ Although longer treatment may potentially increase adverse effects or diminish compliance, treatment requiring endoscopic or histological remission has been associated with a lower relapse rate. ${ }^{65} 66$

Adverse effects for all rectal preparations were under-reported, but seemed comparable. Drug costs using a two week treatment regimen were lower for rectal aminosalicylate products than for most corticosteroid preparations. However, longer term studies may be necessary to evaluate further the median time to remission before it can be concluded that 5-ASA enemas are more cost effective than corticosteroids. To facilitate our analysis, we accepted the authors' definitions of disease response and remission. The ability to pool data effectively from several trials is limited by the variability in outcome criteria. As metaanalyses and overviews are updated, it is essential that methods of diagnosis, definitions of active or inactive disease, and criteria for symptomatic, endoscopic, and histological outcomes are standardised. ${ }^{67}$ Increased attention should also be given to the potential confounding influence of proximal disease margin, formulation of delivery vehicle, volume of preparation, and duration of treatment. As the potential for adverse reactions often governs selection among equally effective agents, adverse effects also must be reported more rigorously.

We conclude that treatment with rectal 5-ASA is superior to treatment with rectal corticosteroids in the management of active distal ulcerative colitis. Rectal budesonide seems to be as effective as conventional rectal corticosteroids, but seems to cause less suppression of endogenous cortisol production. Conventional rectal corticosteroids may be regarded as an alternative rectal treatment for active distal ulcerative colitis once aminosalicylates have failed, or in patients allergic to 5-ASA.

1 Truelove SC, Witts LJ. Cortisone in ulcerative colitis: preliminary report on a therapeutic trial. $B M \mathcal{F}^{\prime} 1954 ; 2$ : preliminar.

2 Truelove SC. Treatment of ulcerative colitis with local hydrocortisone. $B M F$ 1956; 2: 1267-72.

3 Mulder CJJ, Tytgat GNJ. Topical corticosteroids in inflammatory bowel disease. Aliment Pharmacol Ther 1993; 7: 125-30.

4 Chapman NJ, Brown ML, Phillips SF, Tremaine WJ, Schroeder KW, Dewanjee MK, et al. Distribution of mesalamine enemas in patients with active distal mesalamine enemas in patients with active
ulcerative colitis. Mayo Clin Proc 1992; 67: 245-8.

5 Tiel-van Buul MMC, Mulder CJJ, van Royen EA Wiltink EHH, Tytgat GNJ. Retrograde spread of mesalazine (5-aminosalicylic acid)-containing enema in mesalazine (5-aminosalicylic acid)-containing enema in
patients with ulcerative colitis. Clin Pharmacokinet 1991; patients with $247-51$.

6 Jay M, Digenis GA, Foster TS, Antonow DR. Retrograde spreading of hydrocortisone enema in inflammatory bowel disease. Dig Dis Sci 1986; 31: 139-44.

7 Campieri M, Lanfranchi GA, Brignola C, Bazzocchi G, Gionchetti $P$, Minguzzi MR, et al. Retrograde spread of 5 -aminosalicylic acid enemas in patients with active ulcerative colitis. Dis Colon Rectum 1986; 29: 108-10.

8 Kruis W, Bull U, Eisenburg J, Paumgartner G. Retrograde colonic spread of sulphasalazine enemas. Scand $\mathcal{F}$ Gastroenterol 1982;17: 933-8.

9 Nymann-Pantelidis M, Nilsson A, Wagner ZG, Borga O. Pharmacokinetics and retrograde colonic spread of budesonide enemas in patients with distal ulcerative colitis. Aliment Pharmacol Ther 1994; 8: 617-22.

10 Swarbrick ET, Loose H, Lennard-Jones JE. Enema volume as an important factor in successful topical corticosteroid treatment of colitis. Proc Royal Soc Med 1974; 67: 753-4.

11 Van Bodegraven AA, Boer RO, Lourens J, Tuynman HARE, Sindram JW. Distribution of mesalazine enemas in active and quiescent ulcerative colitis. Aliment Pharmacol Ther 1996; 10: 327-32.

12 Farthing MJG, Rutland MD, Clark ML. Retrograde spread of hydrocortisone containing foam given intrarectally in of hydrocortisone containing foam given
ulcerative colitis. $B M \Im$ f $1979 ; 2: 822-4$.

13 Hay DJ. Spreading characteristics of proprietary rectal steroid preparations. In: Wilson CG, Hardy JG, eds. Radionuclide imaging in drug research. London: Croom Radionuclide imaging

14 Wood E, Wilson CG, Hardy JG. The spreading of foam and solution enemas. Int $\mathcal{f}$ Pharmaceut 1985; 25: 191-7.

15 Campieri $M$, Corbelli C, Gionchetti P, Brignola $C$, Belluzzi A, Di Febo G, et al. Spread and distribution of 5-ASA colonic foam and 5-ASA enemas in patients with ulcerative colitis. Dig Dis Sci 1992; 37: 1890-7.

16 Wilding IR, Kenyon CJ, Chauhan S, Hooper G, Marshall S, McCracken JS, et al. Colonic spreading of a non-chlorofluorocarbon mesalazine rectal foam enema in patients with quiescent ulcerative colitis. Aliment Pharmacol Ther 1995; 9: 161-6.

17 Williams CN, Haber G, Aquino JA. Double-blind, placebocontrolled evaluation of 5-ASA suppositories in active distal proctitis and measurement of extent of spread using 99m-Tc-labelled 5-ASA suppositories. Dig Dis Sci 1987; 32: 71S-5S. 
18 Jay M, Beihn RM, Digenis GA, Deland FH, Caldwell L, Mlodozeniec AR. Disposition of radiolabelled suppositories in humans. F Pharm Pharmacol 1985; 37: 266-8.

19 Powell Tuck J, Lennard-Jones JE, May CS, Wilson CG, Paterson JW. Plasma prednisolone levels after admin-
istration of prednisolone-21-phosphate as a retention enema in colitis. BMF 1976; 1: 193-5.

20 Lee DAH, Taylor GM, James VHT, Walker G. Plasma prednisolone levels and adrenocortical responsiveness after administration of prednisolone-21-phosphate as a retention enema. Gut 1979; 20: 349-55.

21 Farmer RG, Schumacher OP. Treatment of ulcerative colitis with hydrocortisone enemas: relationship of hydrocortisone absorption, adrenal suppression, and clinical response. Dis Colon Rectum 1970; 13: 355-61.

22 Luman W, Gray RS, Pendek R, Palmer KR. Prednisolone metasulphobenzoate foam retention enemas suppress the hypothalamo-pituitary-adrenal axis. Aliment Pharmacol Ther 1994; 8: 255-8.

23 Cann PA, Holdsworth CD. Systemic absorption from hydrocortisone foam enema in ulcerative colitis. Lancet 1987; i: 922-3.

24 Bianchi Porro G, Prantera C, Campieri M, Petrillo M, Campanini MC, Gionchetti $\mathrm{P}$, et al. Comparative trial of methylprednisolone and budesonide enemas in the treatment of active distal ulcerative colitis. Eur $\mathcal{F}$ Gastroenterol Hepatol 1994; 6: 125-30.

25 Danielsson A, Hellers G, Lyrenas E, Lofberg R, Nilsson A, Olsson $\mathrm{O}$, et al. A controlled randomized trial of budesonide versus prednisolone retention enemas in active distal ulcerative colitis. Scand $\mathcal{f}$ Gastroenterol 1987; 22: $987-92$.

26 Danish Budesonide Study Group. Budesonide enema in distal ulcerative colitis. Scand $\mathcal{F}$ Gastroenterol 1991; 26: distal ulcera

27 Spencer CM, McTavish D. Budesonide: a review of its pharmacological properties and therapeutic efficacy in inflammatory bowel disease. Drugs 1995; 50: 854-72.

28 Brattsand R. Overview of newer glucocorticosteroid preparations for inflammatory bowel disease. Can $\mathcal{f}$ Gastroenterol 1990; 4: 407-14.

29 Marshall JK, Irvine EJ. Rectal aminosalicylate therapy for distal ulcerative colitis: a meta-analysis. Aliment Pharmacol Ther 1995; 9: 293-300.

30 Schimmer BP, Parker KL. Adrenocorticotropic hormone; adrenocortical steroids and their synthetic analogs; inhibitors of the synthesis and actions of adrenocortical hormones. In: Wonsiewica MJ, McCurdy P, eds. hormones. In: Wonsiewica MJ, McCurdy $\mathrm{P}$, eds. therapeutics. New York: McGraw Hill, 1996: 466.

31 DerSimonian R, Laird N. Meta-analysis in clinical trials. Control Clin Trials 1986; 7: 177-80.

32 Bracken MB. Statistical methods for analysis of effects of treatment in overviews of randomized trials. In: Sinclair JC, Bracken MB, eds. Effective care of the newborn infant. Oxford: Oxford University Press, 1992: 15-6.

33 Breslow NE, Day NE (eds). Statistical methods in cancer research. Lyon: International Agency for Research on research. Lyon: Intermati

34 Lennard-Jones JE, Baron JH, Connell AM, Avery Jones F. A double blind controlled trial of prednisolone21-phosphate suppositories in the treatment of idiopathic 21-phosphate suppositories in the

35 Watkinson G. Treatment of ulcerative colitis with topical hydrocortisone hemisuccinate sodium: a controlled trial employing restricted sequential analysis. BMF 1958; 2: 1077-82.

36 Danielsson A, Lofberg R, Persson T, Salde L, Schioler R, Suhr $\mathrm{O}$, et al. A steroid enema, budesonide, lacking or proctitis. Scand $\mathcal{f}$ Gastroenterol 1992; 27: 9-12.

37 Hanauer S, Robinson $M$. Dose ranging study of budesonide enemas in active distal ulcerative colitis [abstract]. Gastroenterology 1995; 108: A832.

38 Hamilton I, Pinder IF, Dickinson RJ, Ruddel WSJ, Dixon MF, Axon ATR. A comparison of prednisolone enemas with low-dose oral prednisolone in the treatment of acute distal ulcerative colitis. Dis Colon Rectum 1984; 27: 701-2.

39 Lennard-Jones JE, Longmore AJ, Newell AC, Wilson CWE, Avery Jones F. An assessment of prednisone, salazopyrin, and topical hydrocortisone hemisuccinate used as outpatient treatment for ulcerative colitis. Gut 1960; 1 217-22.

40 Bianchi Porro G, Ardizzone S, Petrillo M, Fasoli A, Molteni P, Imbesi V. Low Pentasa dosage versus hydrocortisone in the topical treatment of active ulcerative colitis: a randomized double blind study. $A m \boldsymbol{f}$ Gastrocolitis: a randomized do 1995 ; 90: 736-9.

41 Campieri M, Lanfranchi GA, Bazzocchi G, Brignola C, Sarti F, Franzin G, et al. Treatment of ulcerative colitis with high-dose 5-aminosalicylic acid enemas. Lancet 1981; ii: $270-1$.

42 Danish 5-ASA Study Group. Topical 5-aminosalicylic acid versus prednisolone in ulcerative proctosigmoiditis. Dig Dis Sci 1987; 32: 598-602.

43 Farup PG, Hovde O, Halvorsen FA, Raknerud N, sone foam in patients with distal ulcerative colitis. Scand f Gastroenterol 1995; 30: 164-70.
44 Friedman LS, Richter JM, Kirkham SE, DeMonaco HJ, May RJ. 5-Aminosalicylic acid enemas in refractory distal ulcerative colitis: a randomized controlled trial. $A m \mathcal{F}$ Gastroenterol 1986; 81: 412-8.

45 Lee FI, Jewell DP, Mani V, Keighley MRB, Kingston RD, Record $\mathrm{CO}$, et al. A randomised trial comparing mesalazine and prednisolone foam enemas in patients with acute distal ulcerative colitis. Gut 1996; 38: 229-33.

46 Mulder CJJ, Endert E, Van der Heide H, Houthoff HJ, Wiersinga W, Wiltink EHH, et al. Comparison of 5 -aminosalicylic acid $(3 \mathrm{~g})$ and prednisolone phosphate sodium (enemas (30mg) in the treatment of distal ulcerative colitis: a prospective, randomized, double-blind trial. Scand $\mathcal{F}$ Gastroenterol 1988; 23: 1005-8.

47 O'Donnell LJD, Arvind AS, Cameron D, Talbot IC, Jewell DP, Lennard-Jones JE, et al. Double blind, controlled trials of 4-aminosalicylic acid and prednisolone enemas in distal ulcerative colitis. Gut 1992; 33: 947-9.

48 Sharma MP, Duphare HV, Dasarathy S. A prospective randomized double blind trial comparing prednisolone and 4-aminosalicylic acid enemas in acute distal ulcerative colitis. $\mathcal{F}$ Gastroenterol Hepatol 1992; 7: 173-7.

49 Lofberg R, Ostergaard Thomsen O, Langholz E, Schioler R, Danielsson A, Suhr O, et al. Budesonide versus prednisolone retention enemas in active distal ulcerative colitis. Aliment Pharmacol Ther 1994; 8: 623-9.

50 Tarpila S, Turunen U, Seppala K, Aukee S, Pikkarainen $P$ Elomaa I, et al. Budesonide enema in active haemorrhagic proctitis: a controlled trial against hydrocortis

51 Lamers C, Meijer J, Engels L, Bos L, Van Hogezand R, Driesson $\mathrm{W}$, et al. Comparative study of the topically acting glucocorticoid budesonide and 5-aminosalicylic acting glucocorticoid budesonide and 5-aminosalicylic acid enema therapy of proctitis and proct
[abstract]. Gastroenterology 1991; 100: A223.

52 Lemann M, Galian A, Rutgeerts P, van Heuverzwijn R, Cortot A, Viteau JM, et al. Comparison of budesonide and 5-aminosalicylic acid enemas in active distal ulcerative colitis. Aliment Pharmacol Ther 1995; 9: 557-62.

53 Cobden I, Al-Mardini H, Zaitoun A, Record CO. Is topical therapy necessary in acute distal ulcerative colitis? Double-blind comparison of high-dose oral mesalazine versus steroid enemas in the treatment of active distal ulcerative colitis. Aliment Pharmacol Ther 1991; 5: 513-22.

54 Grace $\mathrm{RH}$, Gent AE, Hellier MD. Comparative trial of sodium cromoglycate enemas with prednisolone enemas
in the treatment of ulcerative colitis. Gut 1987; 28: in the

55 Halpern Z, Sold O, Baratz M, Konikoff F, Halak A, Gilat T. $A$ controlled trial of beclomethasone versus betamethasone enemas in distal ulcerative colitis. $\mathcal{F}$ Clin Gastroenterol 1991; 13: 38-41.

56 Hanauer SB, Kirsner JB, Barrett WE. The treatment of leftsided ulcerative colitis with tixocortol pivalate [abstract]. Gastroenterology 1986; 90: A1449.

57 Lennard-Jones JE. Betamethasone 17-valerate and prednisolone 21-phosphate retention enemas in proctocolitis. nisolone 21-phosphate

58 Mulder CJJ, Endert E, Van der Heide H, Houthoff HJ, Wiersinga W, Wiltink EHH, et al. Comparison of beclomethasone diproprionate ( 2 and $3 \mathrm{mg}$ ) and prednisolone sodium phosphate enemas $(30 \mathrm{mg}$ ) in the treatment of ulcerative proctitis: an adrenocortical approach. Neth $\mathcal{f}$ Med 1989; 35: 18-24.

59 Mulder CJ, Fockens P, Van der Heide H, Tytgat GNJ. A controlled randomized trial of beclomethasone dipropionate ( $3 \mathrm{mg}$ ) versus 5-ASA ( $1 \mathrm{~g}$ ) versus the combination of both $(3 \mathrm{mg} / \mathrm{lg})$ as retention enemas in active distal ulcerative colitis [abstract]. Gastroenterology 1994; 106: A739.

60 Riley SA, Gupta I, Mani V. A comparison of sucralfate and prednisolone enemas in the treatment of active
distal ulcerative colitis. Scand $\mathcal{f}$ Gastroenterol 1989; 24: distal ul.

61 Ruddell WSJ, Dickinson RJ, Dixon MF, Axon ATR. Treatment of distal ulcerative colitis (proctosigmoiditis) in relapse: comparsion of hydrocortisone enemas and rectal hydrocortisone foam. Gut 1980; 21 : 885-9.

62 Van der Heide H, Van den Brandt-Gradel, Tytgat GNJ, Endert E, Wiltink EHH, Schipper MEI, et al. Comparison of beclomethasone dipropionate and prednisolone 21-phosphate enemas in the treatment of ulcerative proctitis. $\mathcal{F}$ Clin Gastroenterol 1988; 10: 169-72.

63 Van Outryve M, Huble F, Van Eeghem P, De Vos $M$ Comparison of ridogrel versus prednisolone, both administered rectally, for the treatment of active ulcerative ministered rectally, for the treatment of active ulcerativ

64 Ilnycki A, Shanahan F, Anton PA, Cheang M, Bernstein CN. The placebo response in ulcerative colitis Bernstein CN. The placebo response in ulcera
[abstract]. Gastroenterology 1996; 110: A929.

65 Meyers S, Janowitz HD. The natural history of ulcerative colitis: an analysis of the placebo response. $\mathcal{F}$ Clin Gastroenterol 1989; 11: 33-7.

66 Riley SA, Mani V, Goodman MJ, Dutt S, Herd ME Microscopic activity in ulcerative colitis: what does it mean? Gut 1991; 32: 174-8.

67 Irvine EJ. Assessing outcome in randomized clinical trials: inflammatory bowel disease. Can $\mathcal{F}$ Gastroenterol 1993; 7 : 561-7. 